\title{
Effect of food additives on deoxynivalenol (DON) reduction and quality attributes in steamed-and-fried instant noodles
}

\begin{abstract}
The effect of food additives reduction on quality attributes in steamed-and-fried instant noodles was investigated. Three additives, 1-ascorbic acid, l-cysteine, and sodium bisulfite, were evaluated for their effect on deoxynivalenol (DON) reduction and sensory acceptability of instant noodles. After screening the different concentrations of three food additives, the maximum reduction of DON was optimized for the following concentration ranges: 1ascorbic acid, $0-100 \mu \mathrm{g} / \mathrm{g}$; 1-cysteine, $0-300 \mu \mathrm{g} / \mathrm{g}$; and sodium bisulfite, $0-200 \mu \mathrm{g} / \mathrm{g}$. The experimental results were fitted to a second-order polynomial model, which gave a coefficient of determination (R2) of 0.987 . The results indicated that the overall optimal condition resulting in the maximum DON reduction in instant noodles was obtained at the following combined level: sodium bisulfite, $167 \mu \mathrm{g} / \mathrm{g}$; 1-cysteine, $254 \mu \mathrm{g} / \mathrm{g}$; and 1-ascorbic acid, $23 \mu \mathrm{g} / \mathrm{g}$. The sensory evaluation of noodles with the optimal condition of additives showed that the overall acceptability was in the range of "like slightly" and was not significantly $(\mathrm{P}>0.05)$ different from the control sample. The optimized protocol produced a mixture that can reduce DON in instant noodles up to $67 \%$. Modifying the processing of instant noodles by using additives may be useful to reduce the risk of DON exposure via instant noodles.
\end{abstract}

Keyword: Food additives; Steamed-and-fried instant noodles; deoxynivalenol (DON) 